\title{
Is the Induced-membrane Technique Successful for Limb Reconstruction After Resecting Large Bone Tumors in Children?
}

\author{
Frank Fitoussi MD, PhD, Brice Ilharreborde MD, PhD
}

Received: 4 September 2014 / Accepted: 20 January 2015 / Published online: 30 January 2015

(C) The Association of Bone and Joint Surgeons $\mathbb{R} 2015$

\begin{abstract}
Background Resection of primary malignant tumors often creates large bony defects. In children, this creates reconstructive challenges, and many options have been described for limb salvage in this setting. Studies have supported the use of an induced-membrane technique after placement of a cement spacer to aid in restoration of bone anatomy.

Questions/purposes We asked: (1) What complications are associated with the induced-membrane technique? (2) How often is bone healing achieved after resection greater than $15 \mathrm{~cm}$ using this technique? (3) What is the functional outcome of patients treated with this technique?
\end{abstract}

Each author certifies that he or she, or a member of his or her immediate family, has no funding or commercial associations (eg, consultancies, stock ownership, equity interest, patent/licensing arrangements, etc) that might pose a conflict of interest in connection with the submitted article."

All ICMJE Conflict of Interest Forms for authors and Clinical Orthopaedics and Related Research ${ }^{\mathbb{B}}$ editors and board members are on file with the publication and can be viewed on request.

Each author certifies that his or her institution approved the human protocol for this investigation, and that all investigations were conducted in conformity with ethical principles of research, and that informed consent for participation in the study was obtained.

This work was performed at Robert Debre Hospital and at Trousseau Hospital, Paris, France.

\section{F. Fitoussi ( $\square)$}

Pediatric Orthopedic and Reconstructive Surgery, Trousseau

Hospital, Paris 6 University, Paris, France

e-mail: franck.fitoussi@trs.aphp.fr

\section{B. Ilharreborde}

Department of Paediatric Orthopaedics, Robert Debre Hospital, Paris, France
Methods We performed a retrospective evaluation of eight patients with a mean age of 13.3 years (range, 11-17 years) treated for a malignant bone tumor between 2002 and 2012 at our centers. The primary malignant tumors involved the proximal humerus, femur, and tibia. All patients were treated using the induced-membrane technique after a resection with mean bone loss of $18 \mathrm{~cm}$ (range, 16-23 cm). The general indication for using the induced-membrane technique during this time was a large diaphyseal defect after resection of the tumor. In addition to using cancellous graft as with the original technique, in the current patients an autogenous nonvascularized fibula was used to enhance stability. The patients were assessed at the last followup using the Musculoskeletal Tumor Society (MSTS) scoring system. Mean followup was 47.1 months (range, 24-120 months), and none of the patients were lost to followup before 2 years.

Results A total of four unplanned reoperations were performed in these eight patients. A fracture of the reconstruction occurred in three patients and all were treated successfully, two with surgery and one with immobilization. Bone fusion was obtained in all patients within 4 to 8 months (mean, 5.6 months) after the reconstruction. The mean healing index was $0.31 \mathrm{month} / \mathrm{cm}$ of reconstruction (range, $0.23-0.5 \mathrm{month} / \mathrm{cm}$ ). At last followup, the mean MSTS score was 74\% (range, 67\%-80\%). Conclusions Our findings suggest that the modified induced-membrane technique is a reasonable alternative to other limb reconstruction techniques for bone tumors in children and has the advantage of not requiring a bone bank or an expensive metal prosthesis. Although more patients will be needed to substantiate our findings, it has become a standard part of our arsenal in the treatment of large bone defects after resection of pediatric primitive bone tumors. Level of Evidence Level IV, therapeutic study. 


\section{Introduction}

Large osseous resections of primary malignant tumors often result in substantial loss of the diaphysis of the involved bone and adjacent musculature. Reconstruction therefore is difficult, and often is accompanied by substantial loss of function.

Studies have supported the use of diaphyseal endoprostheses [1, 14], allografts [5], bone transport [7], vascularized autografts $[8,16]$, or combined use of allografts and vascularized autografts $[18,19]$. The goals of these treatments are to restore the original bone anatomy, provide a construct with the strength to withstand daily use, and potentially provide a mechanism for growth, such as seen with the use of vascularized epiphyseal transfer. However, several major complications have been reported, such as infection, loosening of prostheses, nonunion, fracture, allograft resorption, and donor-site morbidity [12]. Biologic reconstructions usually are preferred in children who have potential for long-term survival. In a previous adult patient series, Masquelet et al. [23] reported the use of a two-stage technique that involved insertion of a cement spacer, induction of a membrane, and subsequent reconstruction of the large bone defect with cancellous bone autograft. Although their series was large [23], the patients had only short-term followup and others [2, 28] have suggested that nonunions and resorption of these grafts might be a problem.

We therefore report on eight patients with primary malignant tumors that required large bony resection greater than $15 \mathrm{~cm}$, who had reconstruction surgery using the induced-membrane technique. We asked the following questions: (1) What are the complications associated with this technique? (2) How often is bone healing achieved after resection greater than $15 \mathrm{~cm}$ using this technique? (3) What is the functional outcome of patients treated with this technique?

\section{Materials and Methods}

Our retrospective study was approved by the local ethics committee, and the procedures were performed between 2002 and 2012 at our centers. Inclusion criteria were that the patient be younger than 18 years, with a primary malignant bone tumor, and an osseous resection greater than $15 \mathrm{~cm}$. Eight patients (mean age, 13 years; range, 11-17 years), who presented with a primary malignant tumor of the proximal humerus (four patients), femur (three patients), or tibia (one patient), were treated using the induced-membrane technique after a large bony resection. There were five male and three female patients. All patients had large local soft tissue masses $(3 \mathrm{~cm}$ to
$10 \mathrm{~cm}$ in diameter) and two presented with pulmonary metastasis. The mean length of the bone defect after resection was $18 \mathrm{~cm}$ (range, 16-23 cm). The patients initially were referred to our department with suspicion of a primary bone tumor. A multidisciplinary team treated them. Preoperative imaging included plain radiographs, bone age, technetium bone scan, local and pulmonary $\mathrm{CT}$, and local MRI. We performed MRI of the entire bone to see the soft tissue and intramedullary extension, and local CT to assess bone osteolysis. Open biopsies always were performed to confirm the diagnosis. Six patients had osteosarcoma and two had Ewing sarcoma. Neoadjuvant chemotherapy was administered according to nationally accepted protocols before and after tumor resection [20].

During the 10-year period when these patients were treated, we treated 32 other patients for similar osseous defects with other approaches, including vascularized fibula and modular endoprostheses. The general indication for using the induced-membrane technique during this time was a large diaphyseal defect after resection of the tumor in children older than 10 years.

No patient has died from his or her disease at this writing, although two patients underwent resections of pulmonary metastases. All patients were available for functional assessment. During the study period, we did only eight of these operations and none of the patients was lost to followup. The mean patient followup was 47.1 months (range, 24-120 months).

\section{Surgical Technique}

All eight patients were operated on using the same principle, a two-stage procedure. The first stage was resection and cement insertion. After the incision was made, excising the skin with the biopsy track, the tumor was dissected, leaving a wide surrounding cuff of normal tissue. The limits of bony resection were identified on preoperative imaging. En bloc resection of the tumor then was performed according to the principles defined by Enneking et al. [10]. In the four patients with proximal humeral resection, an extraarticular resection of the proximal humerus and shoulder was included in the initial resection because glenohumeral joint involvement was seen on preoperative MR images and CT scans. We performed an extrarticular resection and the rotator cuff and part of the deltoid also were resected because of soft tissue tumor extension. The cement spacer was placed in the bed of the resection and initial stabilization was obtained using a nail in six patients and a plate in two patients.

The second stage was reconstruction with autogenous bone graft. The cement-induced membrane most frequently 
forms 6 weeks after the first stage [23]. We delayed (mean, 8.4 months; range, 3-17 months) the bone graft in all of the patients because they needed to complete chemotherapy before reconstruction. The approach was performed with careful opening of the membrane and removal of the cement. The bone was decorticated proximally and distally until bleeding bone was obtained. Autogenous, nonvascularized fibula combined with cancellous iliac grafts from the posterior iliac crest [3] were placed in the induced membrane. The fibula was embedded proximally and distally with a lag screw or wire if needed to enhance the stability inside the membrane. The cancellous bone grafts were placed around the fibula and the membrane was closed in the best way possible. Final stabilization was achieved with a locking plate in five patients and a nonlocking plate in three. In our patients with proximal humeral involvement, reconstructions were performed using a shoulder arthrodesis because of tumor extension in the rotator cuff and deltoid. In the second stage, the proximal extremity of the fusion plate was fixed to the scapular spine (Fig. 1A-E), according to the technique described by Enneking et al. [9, 10].

After humeral reconstructions, the upper arm was immobilized with a thermoplastic thoracobrachial orthosis for 6 weeks. Initial plate stability allowed immediate physiotherapeutic mobilization of the scapula-thoracic joint.

In patients with a lower-limb intervention, passive mobilization of the hip, knee, and ankle was started immediately after the first stage. Touch-down weightbearing was allowed immediately, as the cement spacer improved initial stability. Partial weightbearing was resumed 6 weeks after the bone graft, with full weightbearing after radiologic signs of healing.

Successive radiographs were obtained to observe autograft bone integration. Bone union was considered achieved by surgeons (FF and $\mathrm{BI}$ ) when radiologic signs of bone healing were evident (cortication at the regenerate) and the patient had fully recovered weightbearing capacity or use of the upper limb without pain. We used the ratio (time to bone healing [in months]: length of reconstruct bone [in $\mathrm{cm}$ ]) as our healing index. The patients' function was assessed using the Musculoskeletal Tumor Society (MSTS) scoring system [9], based on six categories. The MSTS score assesses pain, function, and emotional acceptance in patients with upper or lower extremity tumors. For the lower limbs, patients also were evaluated for walking ability, gait, and the use of orthopaedic supports. Patients with upper extremity reconstructions were evaluated for manual dexterity, hand positioning, and lifting ability. Each item of the MSTS system has a maximum of 5 points for the best function. The MSTS score is calculated as a percentage of the maximum possible score. Complications were assessed by the surgeons (FF and BI) involved in the treatment of the patients.

\section{Results}

A total of four unplanned reoperations were performed in these eight patients. Prominence of the shoulder plate of one patient (Patient 2) led to skin ulceration 2 years after bone fusion. The plate was removed without further complications (Fig. 1F, G). One patient (Patient 7) experienced deep infection 3 months after the bone graft. The patient was treated with débridement and antibiotics, with solid union seen 8 months after the reconstruction (Fig. 2). No complication occurred at the donor sites. Spontaneous restitution of the fibula was observed inside the periosteum for all patients except one (Patient 4), the continuity of which was preserved during the intervention (Fig. 3). A fracture of the reconstruction occurred in two patients (Patients 3 and 4) 2 years and 1 year after the second-stage procedure, respectively. Both patients were treated successfully by addition of cancellous bone graft around the fracture site and replacement of the plate (Fig. 4). Two months after the graft procedure, one of the patients (Patient 6), who had humeral reconstruction, had a nondisplaced fracture of the fibular graft related to an intensive physiotherapy regimen. Immobilization with a thermoplastic thoracobrachial orthosis resulted in spontaneous callus formation in 45 days (Fig. 5). There were no local recurrences of the tumors in this series. Donor-site morbidity was seen mainly on the iliac crest with immediate postoperative pain, tenderness, and one hematoma that spontaneously resolved. None of the patients had donorsite morbidity at the fibula.

At the time of this writing, all the patients' grafts had healed (Table 1), with an average healing time of 5.6 months (range, 4-8 months). Cortical bone was observed on all radiographs at latest followup. The mean healing index was 0.31 month per centimeter of reconstruction (range, $0.23-0.5 \mathrm{month} / \mathrm{cm}$ ).

At the most recent followup, the mean MSTS score was $74.25 \%$ (range, $67 \%-80 \%$ ) (Table 2).

\section{Discussion}

Treatment of large segmental bone defects in children is a therapeutic challenge. In patients with primary bone tumors, the goal of treatment is a wide-margin resection that retains a functional limb. Although techniques for treating this type of bone defect have been described [13], biologic reconstructions often are recommended in young patients with the potential for long-term survival $[6,8]$. The use of a two-stage technique with a combination of induced membranes and cancellous autografts to bridge diaphyseal defects up to $25 \mathrm{~cm}$ in length has been reported $[11,22,23,25]$. With this technique, a 

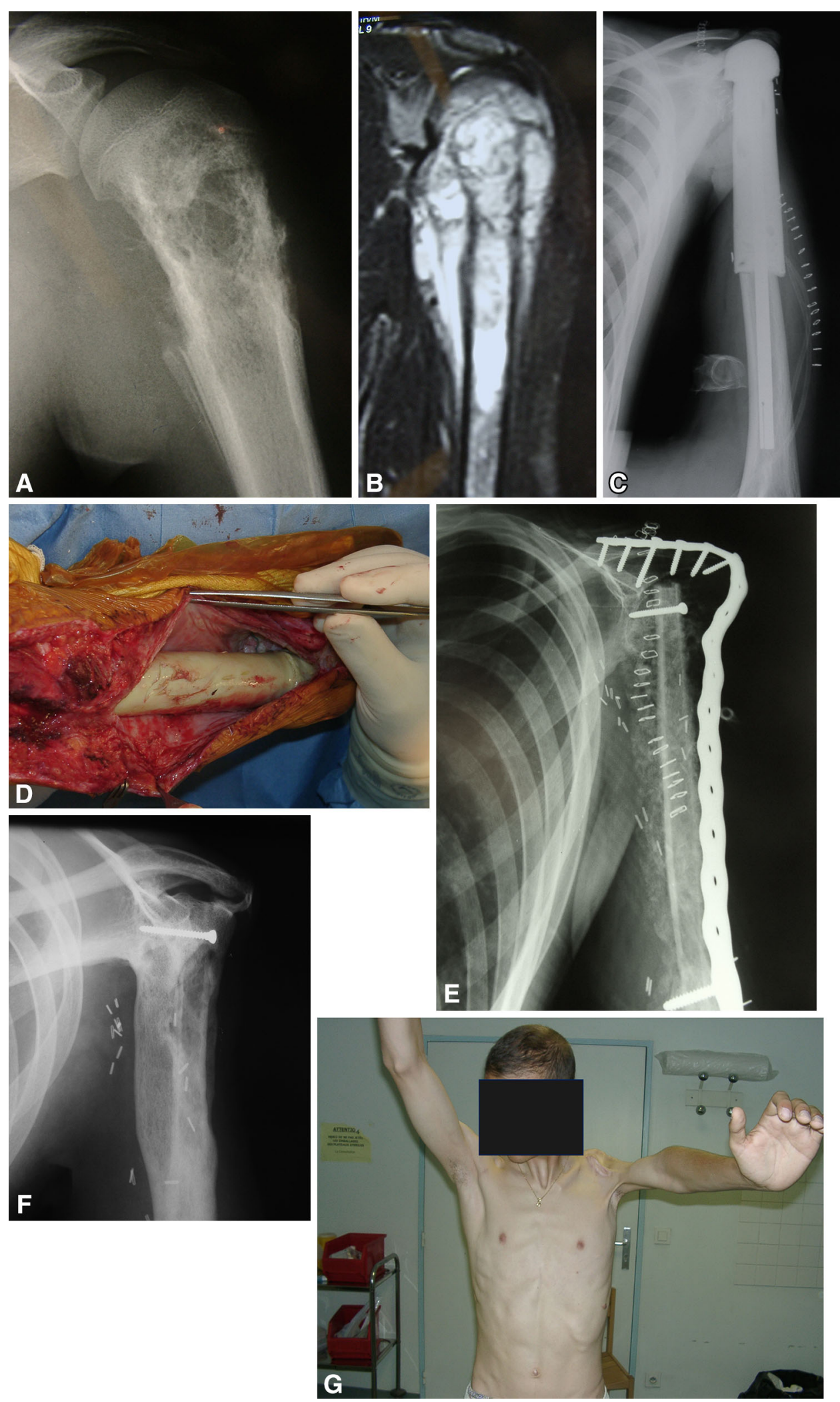
4Fig. 1A-G (A) The AP radiographic view shows an Ewing sarcoma of the proximal left humerus (Patient 2). (B) The MR image shows extension in the surrounding tissues. (C) After large tumor resection, a cement spacer was inserted and stabilized with a nail. (D) During the second stage of the procedure, the membrane was opened carefully before removing the cement. (E) The postoperative AP view of the second stage shows the nonvascularized fibula and the cancellous iliac graft inside the membrane. (F) The AP radiographic view from the 7-year followup after plate removal shows a solid glenohumeral arthrodesis. (G) The patient had no additional complications. He had an MSTS score of $80 \%$.

methylmethacrylate cement spacer induces formation of a membrane in 6 weeks, creating a sheath for cancellous bone autograft. Pelissier et al. [26] showed that these membranes have a rich capillary network and high concentrations of growth and osteoinductive factors with osteogenesis-improving capabilities. The membranous bed prevents resorption of the contained graft and supports revascularization, thus mediating osseous healing (Fig. 5) [26]. However, although the series reported by Masquelet et al. [23] was large, the patients had only short-term followup, and other authors have suggested that nonunions and resorption of these grafts might occur [2, 28]. We therefore sought to evaluate our early experience with the technique, specifically with respect to complications, bone healing, and functional outcomes at a minimum of 2 years.
There were limitations to our study. First, it was retrospective. The series of patients is small and heterogeneous: there were only eight patients with four intercalary and four osteoarticular defects, one involving the tibia, three involving the femur, and four involving the proximal humerus, with arthrodesis reconstruction. The use of the induced-membrane technique in pediatric bone reconstruction after tumor resection is recent and the results we present here are preliminary. Although our study covers a period greater than 10 years, six of the eight patients have 3 or fewer years of followup. Longer time and additional growth of the biological, intercalary segments are necessary to show that our reconstruction technique will provide strong and durable skeletal reconstructions. Additional studies are mandatory.

In our series, the primary complication of the inducedmembrane technique was fracture of the reconstructed bone, which occurred in three patients. However, the outcome in these patients was favorable after placement of additional bone graft around the fracture site, combined with a locking plate in compression in two of the patients. Both patients had bony consolidation after 3 months and no other fracture at last followup. The main point to question here is whether additional fractures will occur in the cohort of patients who achieved healing or if the bone regenerate will hypertrophy sufficiently to prevent future fractures.
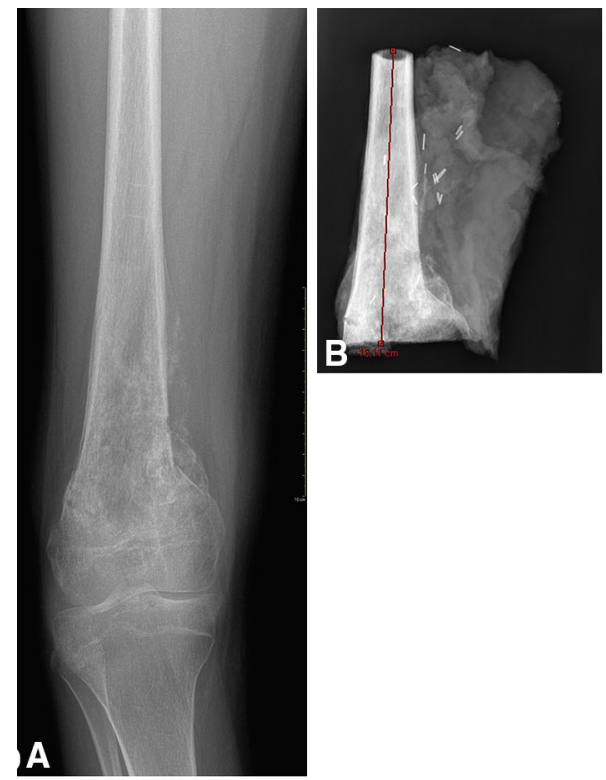

Fig. 2A-E (A) An AP radiographic view shows a right distal femur osteosarcoma (Patient 7). (B) The distal femur resection with epiphyseal preservation can be seen on this radiograph. The red line represents the measurement of the bony resection, which in this case was $16.11 \mathrm{~cm}$. (C) A postoperative lateral radiographic view shows

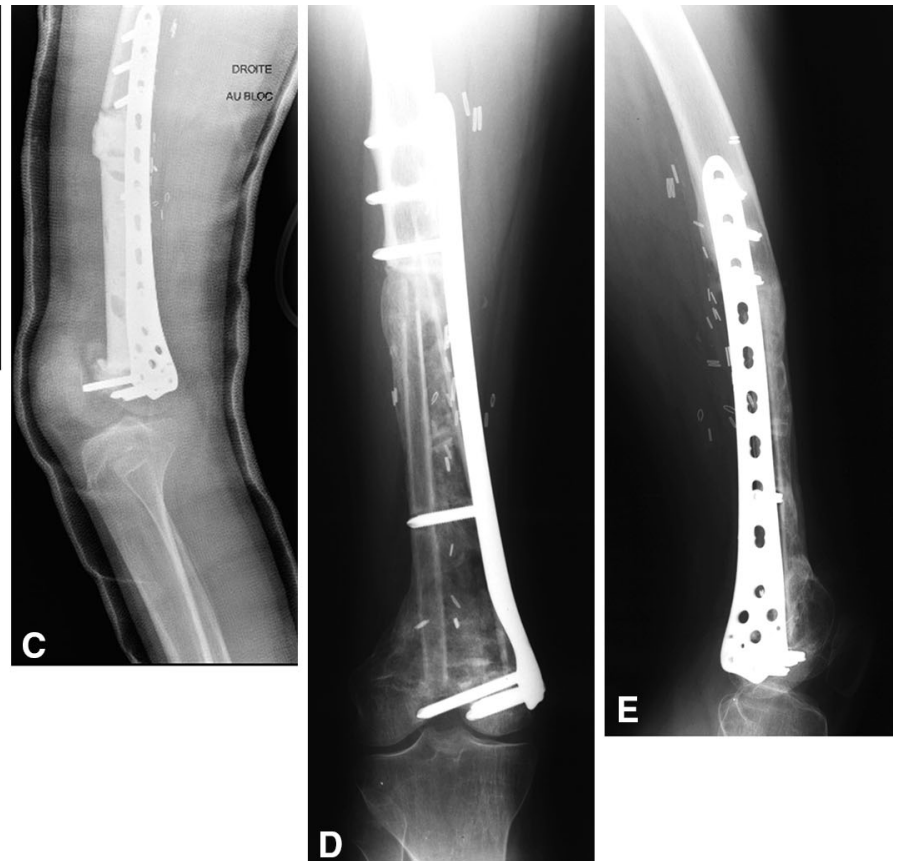

the cement spacer in the bed of the resection stabilized with a locking plate.) (D) AP and (E) lateral radiographic views obtained 2 years after the second stage surgery show the reconstructed femur with the locking plate on the medial side and the nonvascularized fibula on the lateral side of the reconstruction. Knee flexion was $90^{\circ}$. 


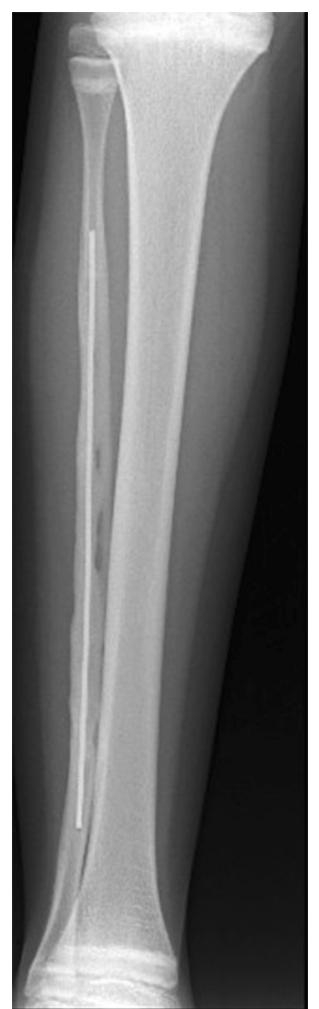

Fig. 3 An AP radiographic view shows spontaneous reconstruction of the fibula on the donor site with intramedullary nailing to stabilize the extremities.
Another reported complication is massive graft resorption after femur reconstruction [2]. None of our patients experienced this complication perhaps because we enhanced stability in the membrane with a nonvascularized fibula. Biau et al. [6] also used a cortical strut harvested from the medial surface of the tibia for a femur reconstruction after Ewing's sarcoma resection, with good stability achieved with a nail. Intercalary segmental bone defects in children most often are repaired using vascularized bone transfer [21], allografts [5], or distraction osteogenesis (the Ilizarov technique) [17]. The Ilizarov technique often is associated with complications, including pin tract infections, segmental alignment problems, and nonunion. Allograft reconstructions are associated with complications such as fracture, nonunion, and infection [15, 24]. The transfer of vascularized bone from the fibula, with or without growth plate [19], is widely used to bridge large defects; however, donor-site morbidity has been reported to occur in as much as $19 \%$ of patients [27]. The relatively poor initial stability in adults owing to the thinness of the fibula has led some surgeons to resort to surrounding the vascularized fibula graft in an allograft shell [19]. Vascularized bone transfer is more suitable for young children in whom initial stability is better, even though some with diaphyseal reconstruction also need a growth-plate transfer. Modular endoprostheses mainly are used in patients with epiphyseal extension of a
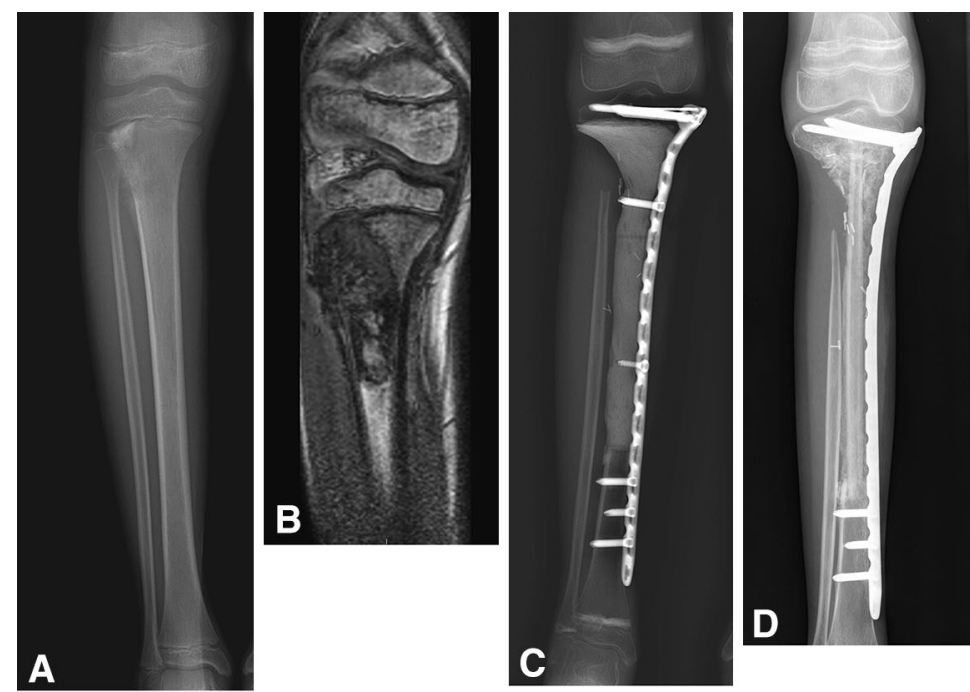

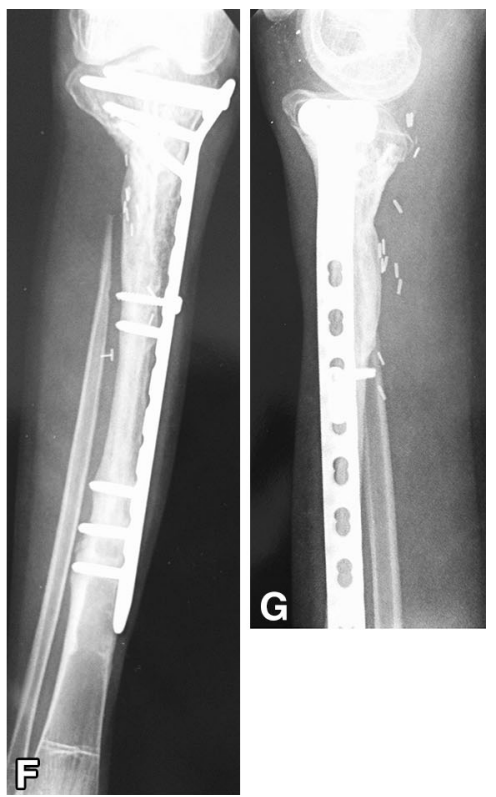

Fig. 4A-G (A) An AP radiographic view shows a proximal tibial osteosarcoma (Patient 3). (B) The MR image shows epiphyseal integrity. (C) An AP radiographic view shows the proximal tibia resection with epiphyseal preservation, stabilized with a locking plate. A cement spacer was placed inside the bed of the resection. Collateral ligaments were reinserted on the epiphysis and patellar ligament on the induced membrane. (D) An AP radiographic view shows the reconstruction 8 months after the second stage surgery. (E) The fracture (in white circle) is seen on this radiograph. The plate was broken at the fracture site. (F) AP and (G) lateral radiographic views were obtained 6 months after cancellous grafting at the fracture site. The plate was changed. Knee flexion was $120^{\circ}$ with active full extension at the last followup. 


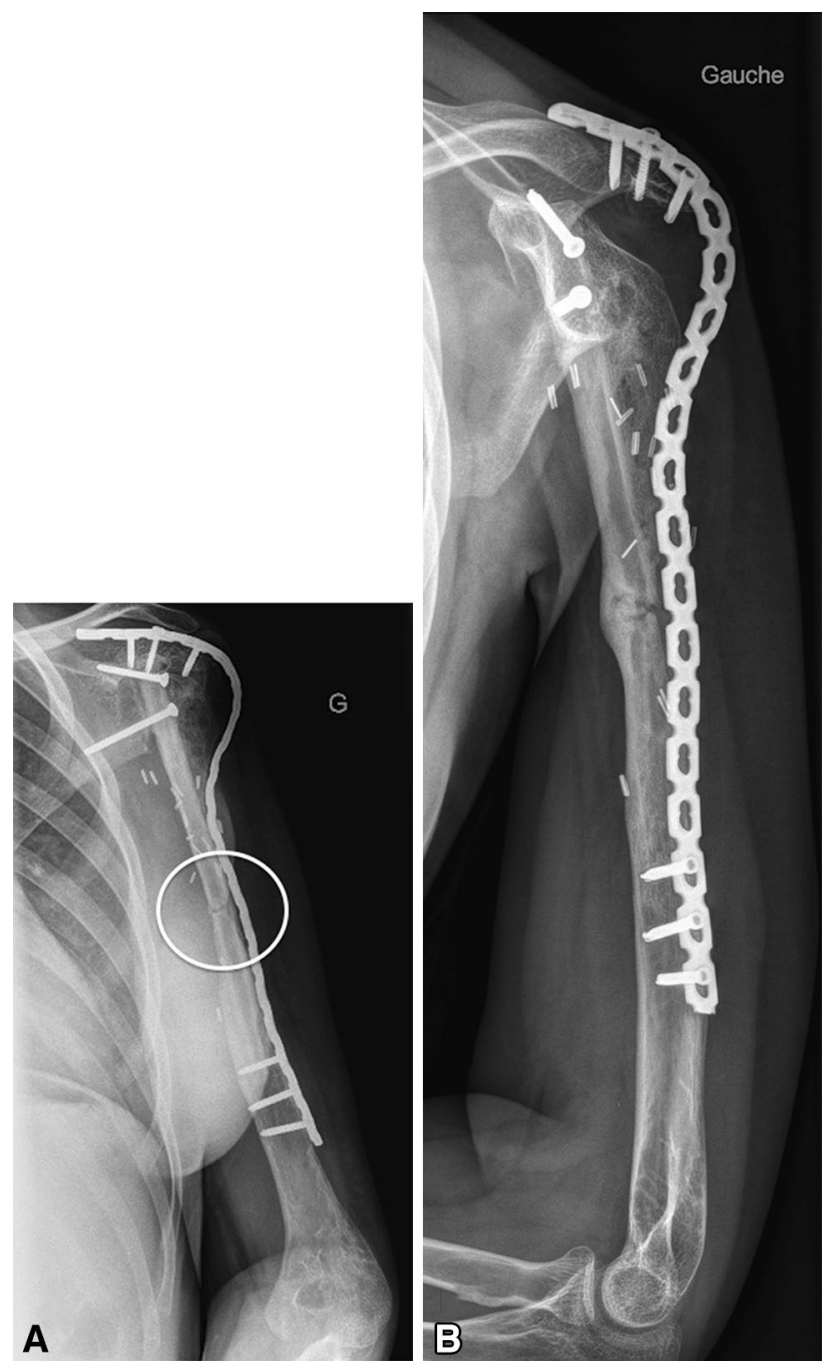

Fig. 5A-B (A) An AP radiographic view obtained 2 months after the second-stage surgery shows a fracture of the fibula (Patient 6). (B) This AP radiographic view shows spontaneous healing with use of a simple thermoplastic thoracobrachial orthosis for 6 weeks. distal femur or proximal tibia tumor. The Masquelet procedure was used after bone tumor resection in a pediatric population [28]. Eleven patients who had reconstruction of the lower limb achieved weightbearing 4.1 months after the second stage of the procedure despite numerous complications such as nonunion and fracture [28]. The differences between that study [28] and our series in terms of complications can be explained by the high stability of our reconstruction technique with the use of a fibula support inside the membrane and a locking plate outside the membrane.

In our study patients, the mean healing time of 5.6 months was short given the large size of the resections, possibly because of a modification of the original technique. Various healing times ranging from 4 to 8 months have been reported for the induced-membrane technique $[23,28]$. In addition to the cancellous graft used in the original technique, the current modification called for insertion of an autogenous nonvascularized fibula inside the membrane as well. The fibula was embedded proximally and distally to enhance stability inside the membrane, which is necessary for bone fusion, especially in large segmental defects. Autologous cancellous bone grafts often are not sufficient to fill a large gap and the nonvascularized fibula has to be preferred to allogenic bone graft in children. The stabilization was augmented with a plate in every patient. In the four patients who required humeral reconstruction, the proximal location of the tumor resulted in sacrifice of the glenohumeral joint during the initial resection. As the four children were all older than 11 years, reconstruction with a shoulder arthrodesis was performed. If these children had been younger than 10 years, we instead would have used a vascularized fibula, including the epiphysis for proximal humeral reconstruction to avoid severe length discrepancy. Femoral tumor resections were performed with a medial approach; the plates

Table 1. Patients' data

\begin{tabular}{|c|c|c|c|c|c|c|c|c|c|c|}
\hline $\begin{array}{l}\text { Patient } \\
\text { number }\end{array}$ & Sex & $\begin{array}{l}\text { Age } \\
\text { (years) }\end{array}$ & Localization & Diagnosis & $\begin{array}{l}\text { Length of } \\
\text { bone resection } \\
(\mathrm{cm})\end{array}$ & $\begin{array}{l}\text { Followup } \\
\text { (months) }\end{array}$ & $\begin{array}{l}\text { Initial } \\
\text { stabilization }\end{array}$ & $\begin{array}{l}\text { Time before } \\
\text { graft (months) }\end{array}$ & $\begin{array}{l}\text { Final } \\
\text { stabilization }\end{array}$ & $\begin{array}{l}\text { Healing } \\
\text { time } \\
\text { (months) }\end{array}$ \\
\hline 1 & M & 11 & Humerus & Osteosarcoma & 17 & 21 & Nancy Nails ${ }^{\mathrm{TM}}$ & 7 & Plate & 5 \\
\hline 2 & M & 15 & Humerus & Ewing sarcoma & 17 & 84 & Nail & 11 & Plate & 4 \\
\hline 3 & M & 10 & Tibia & Osteosarcoma & 19 & 36 & Locking plate & 9 & Locking plate & 8 \\
\hline 4 & M & 17 & Humerus & Ewing sarcoma & 17 & 120 & Nail & 6 & Plate & 4 \\
\hline 5 & F & 13 & Femur & Osteosarcoma & 23 & 30 & Nail & 17 & Locking plate & 8 \\
\hline 6 & $\mathrm{~F}$ & 15 & Humerus & Osteosarcoma & 16 & 26 & Nancy Nails $^{\mathrm{TM}}$ & 6 & Locking plate & 4 \\
\hline 7 & $\mathrm{~F}$ & 14 & Femur & Osteosarcoma & 16 & 24 & Locking plate & 8 & Locking plate & 8 \\
\hline 8 & M & 12 & Femur & Osteosarcoma & 17 & 36 & Locking plate & 3 & Locking plate & 4 \\
\hline
\end{tabular}

The Nancy Nail ${ }^{\mathrm{TM}}$, DePuy Orthopaedics Inc, Warsaw, IN, USA. 
Table 2. Musculoskeletal Tumor Society scores

\begin{tabular}{|c|c|c|c|c|c|c|c|}
\hline Patient number & Pain & Function & Emotional & Hand positioning & Manual dexterity & Lifting ability & MSTS score $(\%)$ \\
\hline \multicolumn{8}{|l|}{ Upper extremity } \\
\hline 1 & 4 & 3 & 5 & 3 & 5 & 4 & 80 \\
\hline 2 & 5 & 3 & 4 & 3 & 5 & 4 & 80 \\
\hline 4 & 3 & 3 & 4 & 3 & 5 & 3 & 70 \\
\hline \multirow[t]{2}{*}{6} & 4 & 3 & 2 & 3 & 5 & 3 & 67 \\
\hline & & & & Supports & Walking & Gait & \\
\hline \multicolumn{8}{|l|}{ Lower extremity } \\
\hline 3 & 4 & 2 & 3 & 4 & 3 & 4 & 67 \\
\hline 5 & 4 & 2 & 4 & 5 & 4 & 3 & 73 \\
\hline 7 & 5 & 3 & 4 & 4 & 4 & 3 & 77 \\
\hline 8 & 5 & 2 & 3 & 5 & 5 & 4 & 80 \\
\hline
\end{tabular}

MSTS = Musculoskeletal Tumor Society.

therefore were placed on the medial side of the reconstruction because of ease of application and to withstand potential adductor muscle deforming forces. The fibula was placed on the lateral side of the reconstruction to enhance stability (Fig. 2D). The main disadvantage of the modified induced-membrane technique is that it is performed in two stages, with the potential associated risks related to the second anesthesia and surgery. According to Masquelet et al. [22], the second stage can be performed after 6 weeks. Previous studies have reported that the optimal timing for the active membrane effectiveness is 4 to 6 weeks after placement of the antibiotic cement implant, with 1 month-old membrane having the highest expression of VEGF. Two-month-old membranes expressed less than $40 \%$ of the VEGF levels $[4,26]$. However, this time frame was not possible in the children receiving neoadjuvant chemotherapy, and although we had to delay the bone graft (mean, 8.4 months), all our patients showed bone healing after a mean of 5.6 months. There was no clear correlation between the timing of the bone graft and bone healing. Another disadvantage encountered was the relatively limited amount of available iliac cancellous bone in the youngest patients. For large diaphyseal reconstruction in young children, we believe that a vascularized fibula transfer is more suitable. The long period of nonfullweightbearing also can be a problem. However, we allowed touchdown weightbearing immediately after the first stage as the cement spacer improved initial stability.

Using the MSTS functional assessment we found that this technique led to satisfactory function in all patients. Other studies with different techniques for reconstruction of bone defects showed less favorable results but they mostly had adult populations [1, 18].

In our experience, the two-stage reconstruction technique has several advantages. Reconstruction of segmental bone defects greater than $15 \mathrm{~cm}$ is now feasible, improving the chance of full tumor resection. Under these conditions, reconstruction is quick and safe. Without specialized equipment required, surgeons with varying experience can perform this straightforward procedure. It is a biologic reconstruction with autogenous bone graft, with no need for prostheses or allographs, reducing the risk of long-term complications. None of our patients with an Ewing tumor had resection with close margins. However, we likely would use radiation therapy if resection with a close margin occurred which might compromise healing. Although more studies evaluating the technique are needed, we consider the modified two-stage technique a reasonable approach in the treatment of large bone defects, although other techniques such as distraction osteogenesis and vascularized bone transfer also are useful. We have used this technique successfully in various other situations such as trauma, infection, and congenital defects. The indications are reserved for large diaphyseal reconstructions after tumor resections in children older than 10 years, and results from the modified induced-membrane procedure seem comparable to those of other techniques used for reconstruction of bone defects after tumor resection.

\section{References}

1. Abudu A, Carter SR, Grimer RJ. The outcome and functional results of diaphyseal endoprostheses after tumour excision. J Bone Joint Surg Br. 1996;78:652-657.

2. Accadbled F, Mazeau P, Chotel F, Cottalorda J, Sales de Gauzy J, Kohler R. Induced-membrane femur reconstruction after resection of bone malignancies: three cases of massive graft resorption in children. Orthop Traumatol Surg Res. 2013;99:479-483.

3. Ahlmann E, Patzakis M, Roidis N, Shepherd L, Holtom P. Comparison of anterior and posterior iliac crest bone grafts in terms of harvest-site morbidity and functional outcomes. $J$ Bone Joint Surg Am. 2002;84:716-720. 
4. Aho OM, Lehenkari P, Ristiniemi J, Lehtonen S, Risteli J, Leskelä HV. The mechanism of action of induced membranes in bone repair. J Bone Joint Surg Am. 2013;95:597-604.

5. Alman BA, De Bari A, Krajbich JI. Massive allografts in the treatment of osteosarcoma and Ewing sarcoma in children and adolescents. J Bone Joint Surg Am. 1995;77:54-64.

6. Biau DJ, Pannier S, Masquelet AC, Glorion C. Case report: reconstruction of a $16-\mathrm{cm}$ diaphyseal defect after Ewing's resection in a child. Clin Orthop Relat Res. 2009;467:572-577.

7. Dormans JP, Ofluoglu O, Erol B, Moroz L, Davidson RS. Case report: reconstruction of an intercalary defect with bone transport after resection of Ewing's sarcoma. Clin Orthop Relat Res. 2005;434:258-264.

8. El-Gammal TA, El-Sayed A, Kotb MM. Reconstruction of lower limb bone defects after sarcoma resection in children and adolescents using free vascularized fibular transfer. J Pediatr Orthop B. $2003 ; 12: 233-243$.

9. Enneking WF, Dunham W, Gebhardt MC, Malawar M, Pritchard DJ. A system for the functional evaluation of reconstructive procedures after surgical treatment of tumors of the musculoskeletal system. Clin Orthop Relat Res. 1993;286:241-246.

10. Enneking WF, Spanier SS, Goodman MA. A system for the surgical staging of musculoskeletal sarcoma. Clin Orthop Relat Res. 1980;153:106-120.

11. Freund R, Wolff TW, Freund B. Silicone block interposition for traumatic bone loss. Orthopedics. 2000;23:795, 799, 802, 804.

12. Friedrich JB, Moran SL, Bishop AT, Wood CM, Shin AY. Free vascularized fibular graft salvage of complications of long-bone allograft after tumor reconstruction. J Bone Joint Surg Am. 2008;90:93-100.

13. Fuchs B, Ossendorf C, Leerapun T, Sim FH. Intercalary segmental reconstruction after bone tumor resection. Eur J Surg Oncol. 2008;34:1271-1276.

14. Hanna SA, Sewell MD, Aston WJ, Pollock RC, Skinner JA, Cannon SR, Briggs TW. Femoral diaphyseal endoprosthetic reconstruction after segmental resection of primary bone tumours. J Bone Joint Surg Br. 2010;92:867-874.

15. Hornicek FJ, Gebhardt MC, Tomford WW, Sorger JI, Zavatta M, Menzner JP, Mankin HJ. Factors affecting nonunion of the allograft-host junction. Clin Orthop Relat Res. 2001;382:87-98.

16. Hsu RW, Wood MB, Sim FH, Chao EY. Free vascularised fibular grafting for reconstruction after tumour resection. J Bone Joint Surg Br. 1997;79:36-42.

17. Ilizarov GA, Ledyaev VI. The replacement of long tubular bone defects by lengthening distraction osteotomy of one of the fragments. 1969. Clin Orthop Relat Res. 1992;280:7-10.
18. Innocenti M, Abed YY, Beltrami G, Delcroix L, Manfrini M, Capanna R. Biological reconstruction after resection of bone tumors of the proximal tibia using allograft shell and intramedullary free vascularized fibular graft: long-term results. Microsurgery. 2009;29:361-372.

19. Jager T, Journeau P, Dautel G, Barbary S, Haumont T, Lascombes P. Is combining massive bone allograft with free vascularized fibular flap the children's reconstruction answer to lower limb defects following bone tumour resection? Orthop Traumatol Surg Res. 2010;96:340-347.

20. Ladenstein R, Pötschger U, Le Deley MC, Whelan J, Paulussen M, Oberlin O, van den Berg H, Dirksen U, Hjorth L, Michon J, Lewis I, Craft A, Jürgens H. Primary disseminated multifocal Ewing sarcoma: results of the Euro-EWING 99 trial. J Clin Oncol. 2010;28:3284-3291.

21. Levin LS. Vascularized fibula graft for the traumatically induced long-bone defect. J Am Acad Orthop Surg. 2006;14:S175-176.

22. Masquelet AC, Begue T. The concept of induced membrane for reconstruction of long bone defects. Orthop Clin North Am. 2010;41:27-37.

23. Masquelet AC, Fitoussi F, Begue T, Muller GP. [Reconstruction of the long bones by the induced membrane and spongy autograft ][in French]. Ann Chir Plast Esthet. 2000;45: 346-353.

24. Ozaki T, Hillmann A, Bettin D, Wuisman P, Winkelmann W. Intramedullary, antibiotic-loaded cemented, massive allografts for skeletal reconstruction: 26 cases compared with 19 uncemented allografts. Acta Orthop Scand. 1997;68:387-391.

25. Pelissier P, Martin D, Baudet J, Lepreux S, Masquelet AC. Behaviour of cancellous bone graft placed in induced membranes. Br J Plast Surg. 2002;55:596-598.

26. Pelissier P, Masquelet AC, Bareille R, Pelissier SM, Amedee J. Induced membranes secrete growth factors including vascular and osteoinductive factors and could stimulate bone regeneration. J Orthop Res. 2004;22:73-79.

27. Vail TP, Urbaniak JR. Donor-site morbidity with the use of vascularized autogenous fibular grafts. J Bone Joint Surg Am. 1996;78:204-211.

28. Villemagne T, Bonnard C, Accadbled F, L'kaissi M, de Billy B, Sales de Gauzy J. Intercalary segmental reconstruction of long bones after malignant bone tumor resection using primary methyl methacrylate cement spacer interposition and secondary bone grafting: the induced membrane technique. J Pediatr Orthop. 2011;31:570-576. 